UCRL-JC-125879

PREPRINT

\title{
LLNL Flash X-ray Radiography Machine (FXR) Double-Pulse Upgrade Diagnostics
}

\author{
M. Ong \\ C. Avalle \\ R. Richardson \\ J. Zentler \\ This paper was prepared for submittal to the \\ 11th IEEE International Pulse Power Conference \\ Baltimore, MD \\ June 29-July 2, 1997
}

June 26, 1997

This is a preprint of a paper intended for publication in a joumal or proceedinga. Since changes may be made before publication, this preprint is made available with the underetanding that it will not be cited or reproduced without the permission of the author. 


\section{DISCLAIMER}

This document was prepared as an account of work sponsored by an agency of the United States Government. Neither the United States Government nor the University of California nor any of their employees, makes any warranty, express or implied, or assumes any legal liability or responsibility for the accuracy, completeness, or usefulness of any information, apparat us, product, or process disdosed, or represents that its use would not infringe privately owned rights. Reference herein to any specific commercial products, process, or service by trade name, trademark, manufacturer, or otherwise, does not necessarily constitute or imply its endorsement, recommendation, or favoring by the United States Government or the University of Californin. The views and opinions of authors expressed herein do not necessarily state or reflect those of the United States Government or the University of California, and shall not be used for advertising or product endorsement purposes. 


\title{
LLNL FLASH X-RAY RADIOGRAPHY MACHINE (FXR) DOUBLE-PULSE UPGRADE DIAGNOSTICS
}

\author{
Mike Ong \\ Carlos Avalle \\ Roger Richardson \\ Jan Zentler \\ Lawrence Livermore National Laboratory \\ Livermore, CA 94550
}

\begin{abstract}
When the FXR machine was first tuned on the 1980's, a minimal amount of diagnostics was available and consisted mostly of power monitors. During the recent accelerator upgrade, additional beam diagnostics were added. The sensor upgrades included beam bugs (resistive wall beam motion sensors) and high-frequency B-dot. Even with this suite of measurement tools, tuning was difficult.

For the current Double-Pulse Upgrade, beam transport is a more complex problem-the beam characteristics must be measured better. Streak and framing cameras, which measure beam size and motions, are being added. Characterization of the beam along the entire accelerator is expected and other techniques will be evaluated also.
\end{abstract}

Each sensor has limitations and only provides a piece of the puzzle. Besides providing more beam data, the set of diagnostics used should be broad enough so results can be cross validated. Results will also be compared to theoretical calculations and computer models, and successes and difficulties will be reported.

\section{Introduction}

Lawrence Livermore National Laboratory's Flash X-Ray machine (FXR) is a tool to radiograph mockups of imploding primary stages of nuclear devices. FXR is an induction linear accelerator specifically designed for diagnosing hydrodynamic tests. An injector introduces an electron beam into the FXR accelerator. (See Figure 1.) After passing through the accelerator, the beam enters a drift section that directs it toward a 1-millimeter-thick strip of tantalum, called a target. As the highenergy electrons pass through the target, the electric field created by the stationary charged particles of the heavy tantalum nuclei causes the electrons to decelerate and radiate some of their energy in the form of $x$-rays. The recently completed upgrade to the FXR improved the quality of the beam. In the near future, LLNL will be adding a double-pulse feature to the FXR to provide two radiographs of a single explosion- implosion separated by 1 to 5 microseconds. ${ }^{1}$

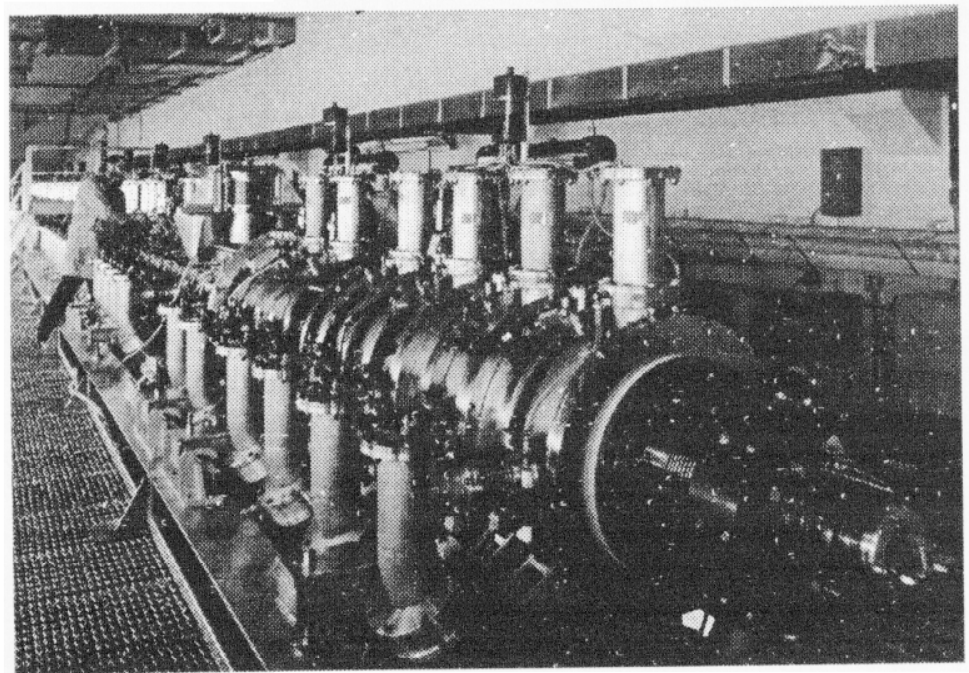

Figure 1. Picture of injector end of the FXR linear induction accelerator.

Work performed under the auspices of the U.S. Department of Energy by the Lawrence Livermore National Laboratory under Contract W-7405-Eng-48. 
This paper provides an overview of the current and evolving FXR beam diagnostics and their relationship to accelerator design and tuning. The objective of the diagnostics is to gather information for improving the quality of the beam. LLNL's figure of merit is $\mathrm{x}$-ray dose divided by spot diameter squared.

Beam characteristics that affect dose include current, duration, and energy. (See Figure 2.) Spot size is determined by energy, position, motion, and beam size. The diagnostic requirements for a production accelerator is different from an experimental machine. The sensors must be compact and non-intrusive. It is unattractive to disassemble the accelerator to add or move diagnostics. The types of diagnostics are listed across the top of the figure. Every cell has a voltage monitor. There are 16 Beam Bug (resistive wall) stations and 4 B-dot stations. ${ }^{2}$ These nonintrusive sensors report the first four beam characteristics.

However, measuring the beam size has been more difficult. We are working on two approaches. First, the diamagnetic loop is being evaluated. It has limitations in a tuning situation where the beam is not well behaved. Second, we plan to insert kapton targets into the accelerator cells and record the light produced by the beam striking the targets. We have both high-speed streak and framing cameras.

Beam Diagnostics

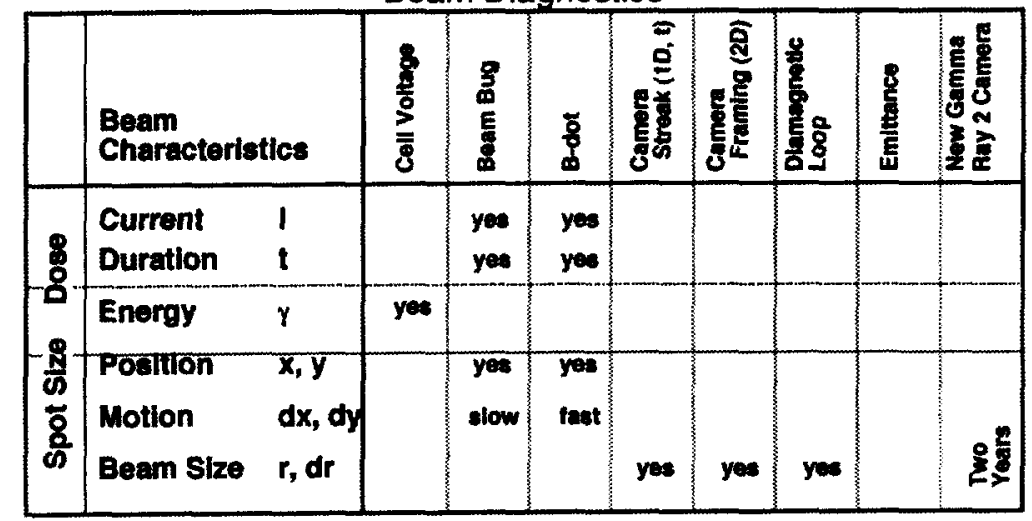

Figure 2. Overview of FXR beam diagnostics.

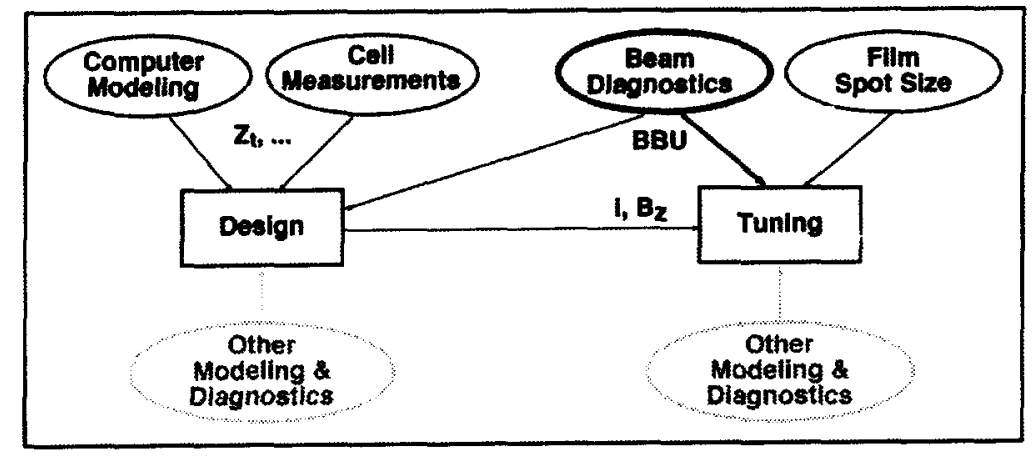

Figure 3. Relationship between diagnostics, design, and tuning.

The role of beam diagnostics is to provide feedback and insight for accelerator design and tuning to optimize the figure of merit. (See Figure 3.) Rather than give performance specifications for the sensors, we will instead explain how the diagnostic information was used to improve the performance of the accelerator. We will focus on the problems of Beam Breakup instability (BBU) and corkscrew. BBU is a crucial problem that had to be overcome with design changes and tuning to achieve better beam performance.

\section{Beam Breakup Instability}

When the beam passes the acceleration gap, it can interact with resonance modes in the cell cavities. ${ }^{3}$ (See Figure 4.) This generates transverse motions in the beam that grows along the accelerator. If shots are poorly tuned, the BBU can expand too much and scrape the beam pipe resulting in current lost. The top plot is a B-dot measurement made at the end of the accelerator. The peak beam centroid motion is about $5 \mathrm{~cm}$, which is large considering the beam pipe radius is $7.3 \mathrm{~cm}$. The measured dominant frequency is $820 \mathrm{MHz}$. For a series of 4 tunes, the effect of BBU on the $x$-ray source spot size can be seen on the left plot. The spot size is determined by analyzing a shadow image on exposed radiographic film. If we could eliminate all cavity induced beam motion, the spot size would still be $1.6 \mathrm{~mm}$. The right plot provides some insight about the uncertainty of the diagnostics. The tune was fixed and 
a number of film spot sizes were measured. The connection between beam motions and cavity resonances is demonstrated in the lower plot. The spectral content of a well tuned shot is shown along with a cavity measurement. The transverse impedance was taken with a network analyzer on a single cell. Higher resistance generally translates to more BBU growth. Note the match at $820 \mathrm{MHz}$.

BBU growth along the accelerator is documented in Figure 5. The beam motion is measured at three locations and transformed into the frequency domain. The $820 \mathrm{MHz}$ component is extremely small at the beginning of the accelerator and can only be seen in the spectral plot. The vertical scale is difficult to interpret and should be read as relative units.

The major design and operating parameters that effect $B B U$ is predicted by the following simplified equation: 4,5

$$
\zeta_{m}=\zeta_{m-1} \mathrm{e}^{\mathrm{kiz} \mathrm{z}_{\mathrm{t}} \mathrm{n} / \mathrm{B}_{\mathrm{z}}}
$$

where $\xi_{m}$ is the average BBU amplitude at a frequency. It is determined by the motion $\left(\xi_{m-1}\right)$ in the previous cell section, the beam current (i), the average transverse resistance $\left(Z_{t}\right)$, the number of cells $(n)$ in a section, and the solenoidal transport field $\left(B_{Z}\right)$. The values predicted by the formula and measurements are shown in Figure 6. The constant (k) was determined with the base line tune. The increased current and field tunes produced BBU results that are close to the predicted values.
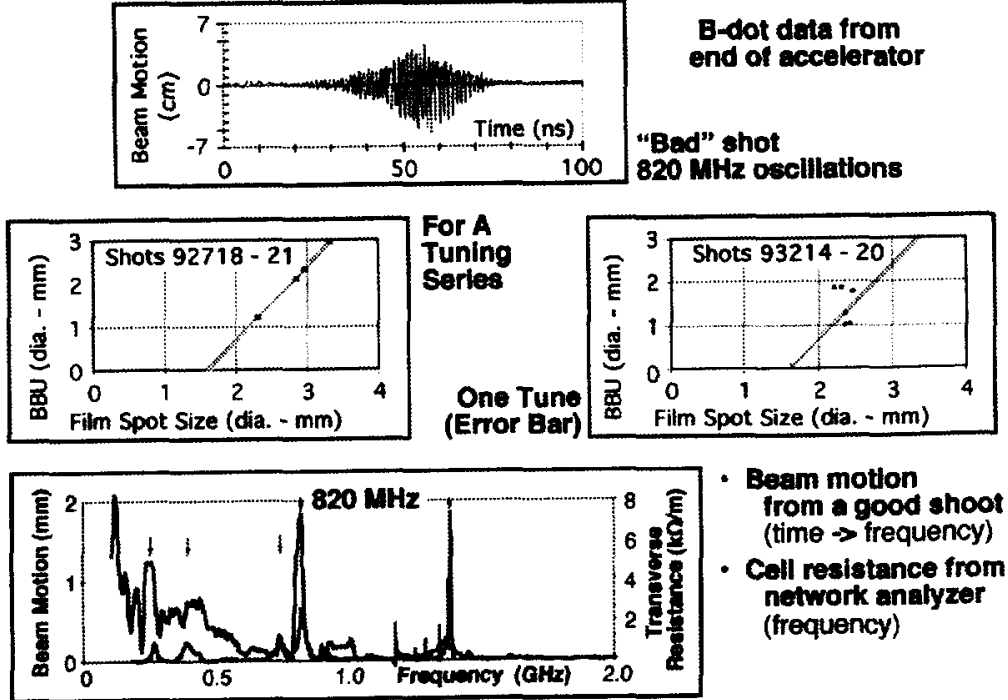

- Beem motion from a good shoot (time $\rightarrow$ frequency)

- Cell resistance from network analyzer (frequency)

Figure 4. Beam Breakup Instability adversely affects $x$-ray spot size.
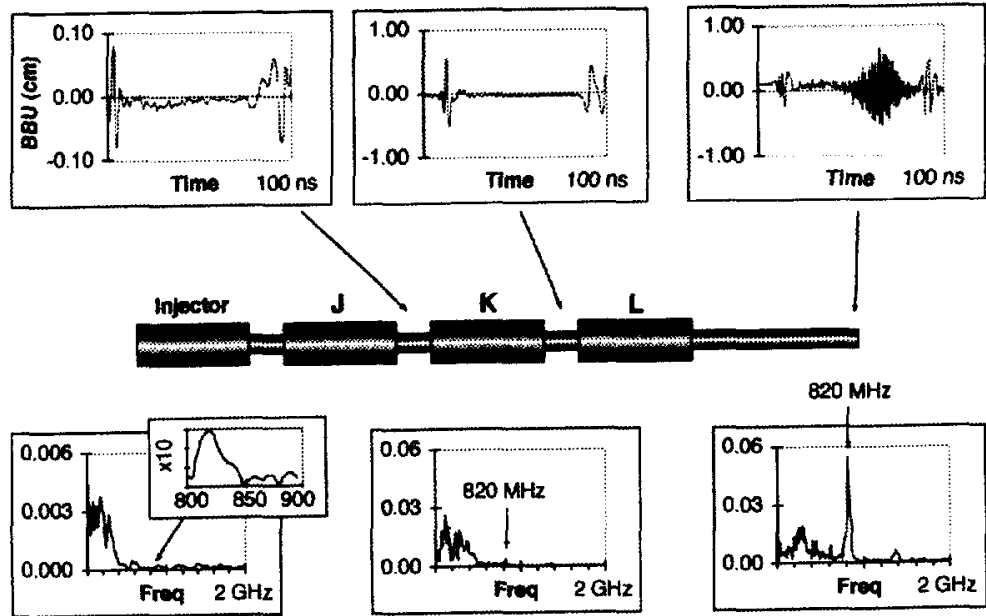

Figure 5. BBU grows along the accelerator.

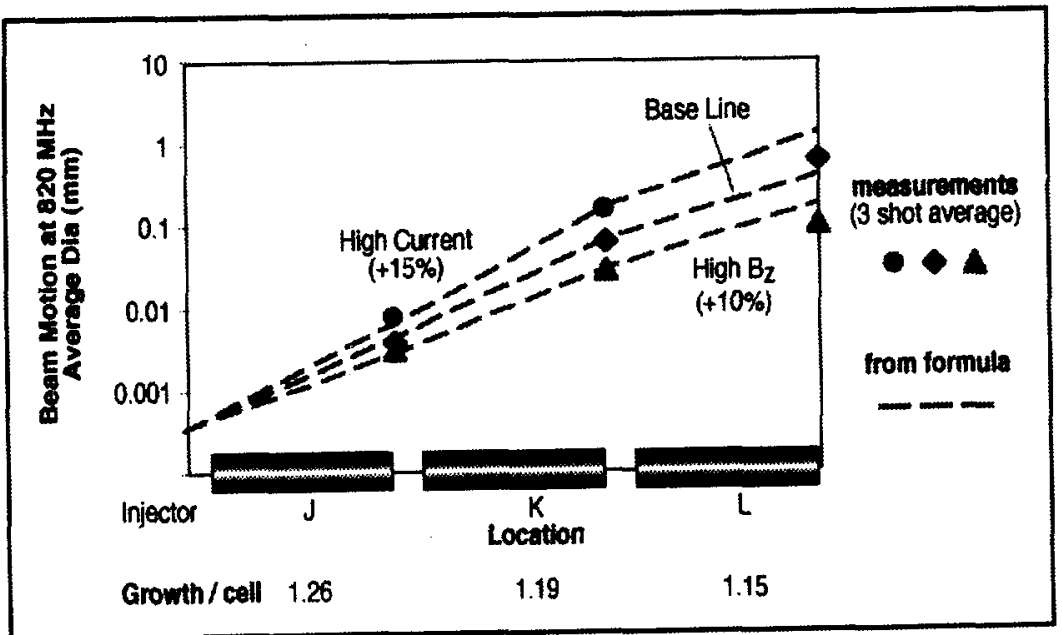

Figure 6. BBU growth is effected by beam current and the focusing field. 


\section{Computer Modeling and Cell Measurements to Suppress BBU}

A number of cell modifications are being considered to reduce the transverse impedance for the upgrades. It is relatively simple to try changes on a single cell, rather than the whole accelerator. Hence, impedance reducing techniques were evaluated on a cell with both computer models and impedance measurements. Figure $7 \mathrm{a}$ shows a cross-section of the cell with the modifications. Figure $7 \mathrm{~b}$ gives an example of the results. A large ferrite toroid was embedded in the end plate that forms a part of the acceleration gap. The first type of ferrite we tested had low loss, and although it lowered the resonant frequency and ' $\mathrm{Q}$ ' by dispersion, it did not absorb much of the energy. Lossier types are being considered. Figure $7 \mathrm{c}$ shows another modification being considered using a thin sheet of RF absorber inside the vacuum housing.

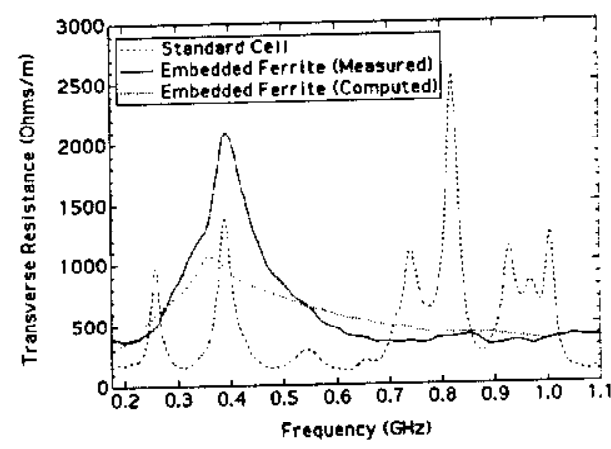

Figure $7 \mathrm{~b}$. Computer simulation and cell measurement of embedded ferrite.

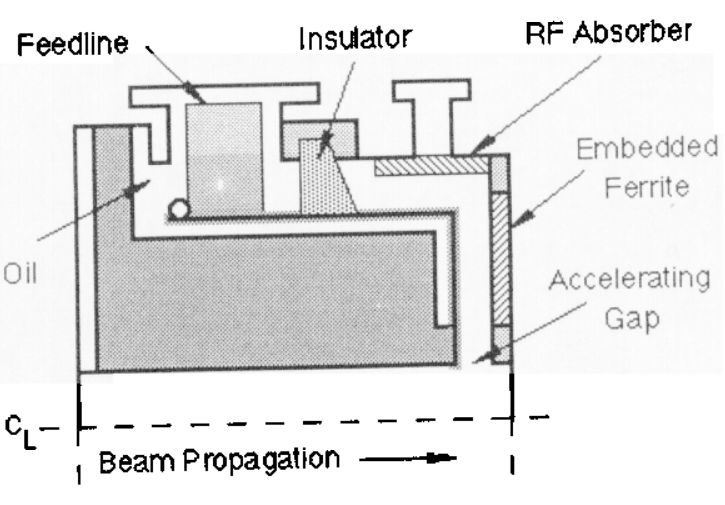

Figure 7a. Cross-section of the FXR cell showing the location of the test materials.

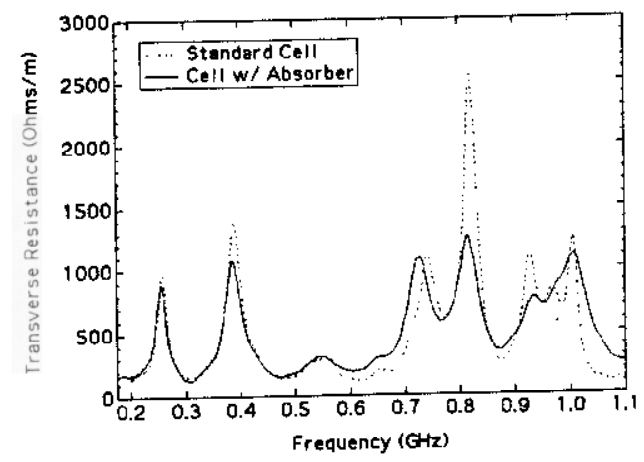

Figure 7c. Impedance measurement of absorbing material.

\section{Controlling Beam Corkscrew Motion}

Corkscrew motion is caused by misalignment of the magnetic focusing field and beam energy variation. It is of lower frequency than BBU. In order to transport the beam and produce a small spot size at the target, corkscrew motion must be minimized. FXR has pairs of steering coils associated with most of focusing magnets which are used to align the beam. Beam centroid motion is measured at these frequencies with resistive wall sensors, commonly known at LLNL as beam bugs ${ }^{2}$. The beam bugs also measure the beam current. There are fewer of these sensors than steering coils.

The steering tune process begins at the injector. In turn, each steering

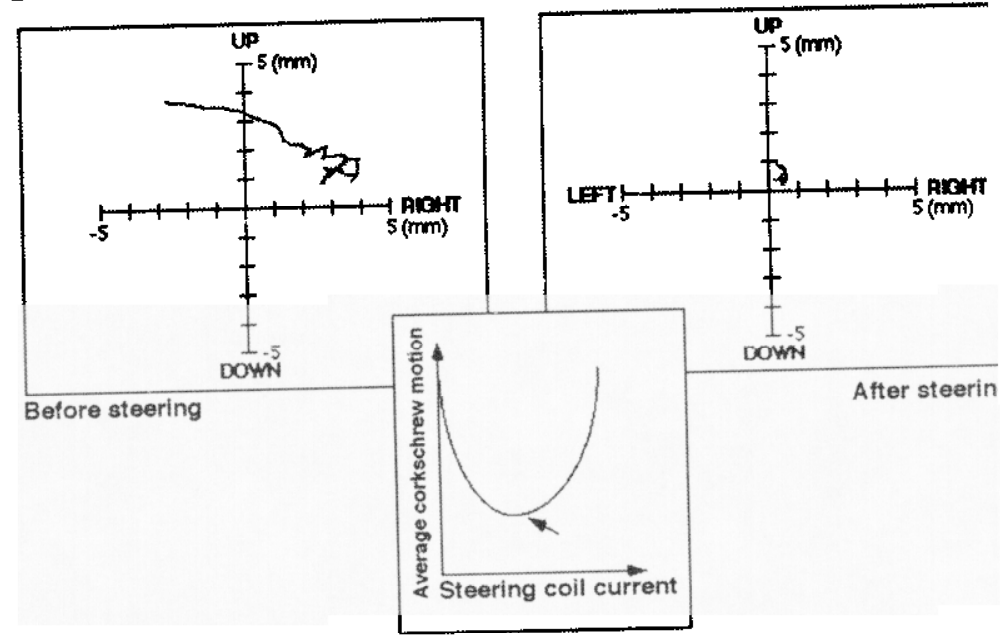

Figure 8. The beam is steered to minimize corkscrew motion as measured by Beam Bugs. 
coil current is varied and the beam moition is recorded by a downstream beam bug. The corkscrew motion is averaged over the beam duration. This time average corkscrew motion becomes a metric which we strive to minimize ${ }^{6}$. Figure 8 shows the low frequency moition of the beam exiting FXR before and after tuning. Also shown is the typical relationship between a steering coil current and corkscrew amplitude immediately downstream of the coil.

\section{Optical Beam Size Diagnostic Development}

The one beam characteristic that is not easily determined is beam size. We currently have a diagnostic cross with viewing ports in the draft section at the end of the accelerator.

A thin piece of kapton at $45^{\circ}$ is inserted in the electron beam to generate optical transition radiation. This radiation allows the beam to be imaged optically. A Hamamatsu streak camera (C1587HR) is focused on the kapton to view the beam size (in one dimension) as a function of time. The camera has a $576 \times 384$ CCD output, however the data in this paper is from digitized photos of a video conversion of the CCD. (See Figure 9.) The temporal and spatial resolution of the camera depends on many factors such as: slit width, sweep time, CCD pixels, magnification, focus and video resolution. For this data the resolution is estimated to be about $1 \mathrm{~mm}$ and $2 \mathrm{~ns}$. The data is taken at a location after the accelerator modules, but before the beam is brought to a final focus.
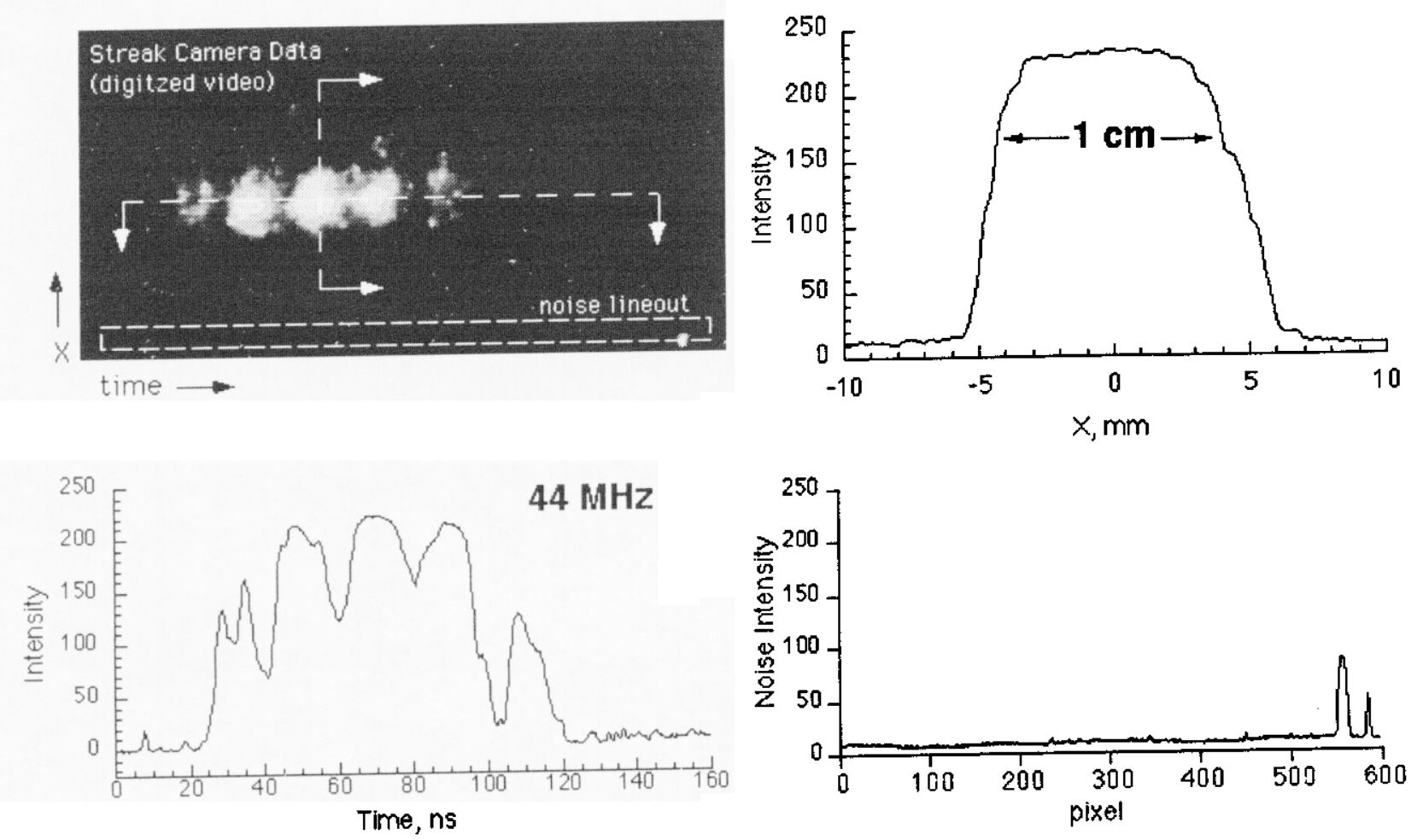

Figure 9. Image of FXR electron beam from steak camera.

A lineout of the data in the spatial direction (an average of around $5 \mathrm{~ns}$ at the peak signal), gives a beam diameter at this location of about $1 \mathrm{~cm}$ FWHM. In the time direction (horizontal in the photograph) it is obvious that the signal is oscillating. A lineout in the temporal direction (a spatial average of about 3 $\mathrm{mm}$ in the middle of the beam) illustrates this more clearly. An FFT indicates that the frequency of oscillation is about $44 \mathrm{MHz}$. This oscillation could be due to a corkscrew motion or periodic tilting of the beam, and needs further investigation.

A series of photographs were taken with tuning parameters varied (steering and focusing magnets). The data is available within seconds of the shot, which illustrates that this diagnostic should facilitate rapid tuning. 
Because of the $820 \mathrm{MHz}$ BBU, a fast 1 ns shutter speed framing camera is also being prepared. The long-term objective is to measure the beam size at many locations along the accelerator. However, there is insufficient room to easily insert a diagnostic window near accelerator cells. We are designing a target probe that can be inserted into the acceleration gap.

\section{Summary}

Beam diagnostics is necessary for tuning the FXR. More importantly, it provides crucial insight for improving the design of the accelerator. While evaluations of single cell design modifications are necessary, only measurement of the accelerated beam produces a complete picture of the improvement. Our suite of diagnostic tools is fairly complete, though, we must still finish development of an optical probe to determine beam size.

\section{Acknowledgment}

We wish to acknowledge the support of Ray Scarpetti, our project leader. John Boyd provided much insight about beam acceleration and transport. We want to thank Rod Kerr for sharing his knowledge about $x$-ray generation and analysis of the film spot size. Scott Nelson modeled the accelerator cell. We wish to thank Rich Gilliam for his mechanical wizardry. We also want to thank Lorna Naugle for artist additions and handling the details.

\section{References}

1. Ray Scarpetti, Jan-Mark Zentler, John Boyd, Greg Early, Rod Kerr, Ron Kihara, Ken Griffin, "Upgrades to the LLNL Flash X-Ray Induction Linear Accelerator (FXR)", 11th IEEE International Pulsed Power Conference, June 29-July 2, 1997.

2. Ken Struve, "Electrical Measurement Techniques for Puised High Current Electron Beams," UCRL93261, Lawrence Livermore National Laboratory, April 1, 1986.

3. Daniel Birx, "Microwave Measurements of the ETA Accelerating Cavity", UCID-18582, Lawrence Livermore National Laboratory, March 12, 1980.

4. V. K. Neil, L. S. Hall, R. K. Cooper, "Further Theoretical Studies of the Beam Breakup Instability", Particle Accelerators, 1979, Vol. 9, pp. 213-222.

5. R. J. Briggs, D. L. Birx, G. J. Caporaso, V. K. Neil, and T. C. Genoni, "Theoretical and Experimental Investigation of the Interaction Impedances and $Q$ Values of the Accelerating Cells in the Advanced Test Accelerator", Particle Accelerators, 1985, Vol. 18, pp. 41-62.

6. Y. J. Chen, "Beam Control in the ETA-II Linear Induction Accelerator", Proc. 1992 Linear Accelerator Conference, Ottowa, Ontario, Canada, 1992, p. 540. 


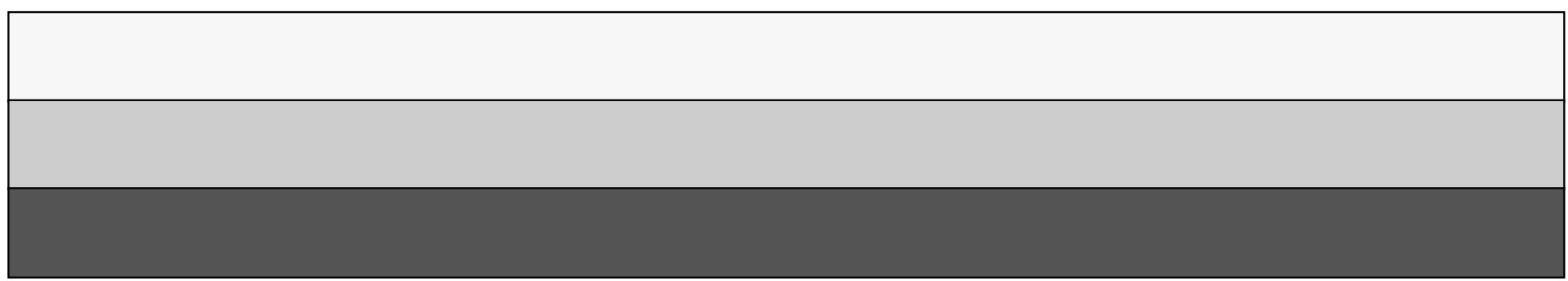

